Wolters Kluwer Lippincott Health Williams \& Wilkins

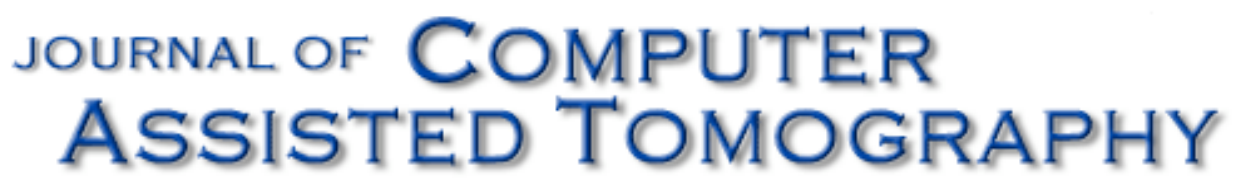

(C) Lippincott-Raven Publishers

Volume 22(1), January/February 1998, pp 153-165

\title{
Automated Image Registration: II. Intersubject Validation of Linear and Nonlinear Models
}

[Image Processing]

\author{
Woods, Roger P.; Grafton, Scott T.; Watson, John D. G.; Sicotte, Nancy L.; \\ Mazziotta, John C.
}

\begin{abstract}
From the Division of Brain Mapping (R. P. Woods, N. L. Sicotte, and J. C. Mazziotta) and Departments of Neurology (R. P. Woods, N. L. Sicotte, and J. C. Mazziotta), Pharmacology (J. C. Mazziotta), and

Radiology (J. C. Mazziotta), UCLA School of Medicine, Los Angeles, CA, and Departments of Neurology and Nuclear Medicine, Emory University School of Medicine, Atlanta, GA (S. T. Grafton), U.S.A.; and

Department of Medicine, University of Sydney, and Neuropsychology Unit, Royal Prince Alfred Hospital, Camperdown, Australia (J. D. G. Watson).

Address correspondence and reprint requests to Dr. R. P. Woods at Department of Neurology, 710 Westwood Plaza, Rm. 1-240, Los Angeles, CA 90095, U.S.A.
\end{abstract}

\section{Abstract}

Purpose: Our goal was to validate linear and nonlinear intersubject image registration using an automated method (AIR 3.0) based on voxel intensity.

Method: PET and MRI data from 22 normal subjects were registered to corresponding averaged PET or MRI brain atlases using several specific linear and nonlinear spatial transformation models with an automated algorithm. Validation was based on anatomically defined landmarks.

Results: Automated registration produced results that were superior to a manual nine parameter variant of the Talairach registration method. Increasing the degrees of freedom in the spatial transformation model improved the accuracy of automated intersubject registration.

Conclusion: Linear or nonlinear automated intersubject registration based on voxel intensities is computationally practical and produces more accurate alignment of homologous landmarks than manual nine parameter Talairach registration. Nonlinear models provide better registration than linear models but are slower.

Registration of tomographic images acquired from different subjects poses unique problems not encountered with intrasubject registration. Biological considerations place few constraints on the choice of an appropriate intersubject spatial transformation model, and the very existence of a fully valid one-to-one mapping is doubtful, even at the macroscopic level (1). Despite such difficulties, intersubject registration tools are routinely used to map imaging data from one subject to another or to a common atlas. These methods allow data to be either compared or pooled across subjects for statistical purposes. When used to map data into a standardized reference space such as the Talairach coordinate space $(2,3)$, these methods also simplify interlaboratory communication and archiving of anatomic locations.

A new method for intersubject registration is described and validated here. The method is part of the Automated Image Registration (AIR 3.0) package. Many other methods have been suggested for manual, automated, or semiautomated intersubject registration (2-11). The new method has the following distinguishing cardinal characteristics: (a) It is general, requiring no explicit identification of anatomic features and allowing arbitrary selection of the registration target; (b) it makes no assumptions about voxel intensities within the brain, making it equally applicable to a variety of data types; (c) aside from removal of the scalp, skull, and dura from images in which these features are prominent, it requires no user intervention; (d) it includes a variety of linear and nonlinear spatial transformation models, allowing systematic comparison and selection of the model most appropriate for a given situation; (e) the spatial transformation models are invariant under affine transformations such as rotation, translation, and linear rescaling; ( $f$ ) it has been validated using anatomic criteria; and $(\mathrm{g})$ it is a fully integrated part of AIR 3.0, which also allows intrasubject registration within and across modalities and is freely available to the research community at the World Wide Web 
Universal Resource Locator (URL):http://bishopw.loni.ucla.edu/AIR3/index.html

\section{REGISTRATION ALGORITHM}

Implementation of intersubject registration in AIR 3.0 is very similar to implementation of intrasubject registration, described separately (12). For linear spatial transformation models, the only difference is the spatial transformation model itself. The spatial transformation models investigated here include the following:

1. Global rescaling linear model. This model adds a single global rescaling parameter to the $6 \mathrm{df}$ required for rigid body rotation and translation. Angles and relative distances are preserved, but absolute distances are not.

2. Nine parameter "Talairach" linear model. This model allows for independent rescaling along each of the three primary coordinate axes of the reference volume in addition to the arbitrary rotation and translation provided by a rigid body model. If the brain represented in the reference volume has been oriented properly with respect to the line between the anterior and posterior commissures (AC-PC line) and the midsagittal plane, the model is mathematically equivalent to a model used to derive stereotaxic Talairach coordinates $(3,6)$. This model does not preserve angles or distances but does have the property that parallel lines will remain parallel. In this sense it can be viewed as a specific case of the more general affine model.

3. Affine linear model. This model has 12 parameters and allows for rotation, translation, and independent rescaling along any arbitrary set of axes. The model does not preserve distances or angles, but lines that were parallel before transformation remain parallel after transformation.

4. Second order nonlinear model. This model computes the coordinate location of a voxel in the reslice file $\left(x^{\prime}, y^{\prime}, z^{\prime}\right)$ as a second order polynomial of its location in the reference file $(x, y, z)$ :EquationThis model has a total of 30 spatial transformation parameters.

$$
\begin{aligned}
x^{\prime}= & P_{x 0}+P_{x 1} x+P_{x 2} y+P_{x 3^{z}}+P_{x 4} x^{2}+P_{x 5} x y+P_{x 6} x z \\
& +P_{x 7} y^{2}+P_{x 8} y z+P_{x 9} z^{2} \\
y^{\prime}= & P_{y 0}+P_{y 1} x+P_{y 2} y+P_{y 3} z+P_{y 4} x^{2}+P_{y 5} x y+P_{y 6} x z \\
& +P_{y 7} y^{2}+P_{y 8} y z+P_{y 9} z^{2} \\
z^{\prime}= & P_{z 0}+P_{z 1} x+P_{z 2} y+P_{z 3} z+P_{z 4} x^{2}+P_{z 5} x y+P_{z 6} x z \\
& +P_{z 7} y^{2}+P_{z 8} y z+P_{z 9} z^{2}
\end{aligned}
$$

Equation 4D

5. Third, fourth, and fifth order nonlinear models. These models are analogous to the second order model but involve higher order polynomials. They have 60, 105, and 168 parameters, respectively.

For linear spatial transformation models, AIR allows all model parameters to be adjusted immediately from the first iteration. For nonlinear models this is not generally advisable: Misalignments that could have been corrected by simple rotations, translations, or rescaling can lead instead to large inappropriate high order deformations. The nonlinear registration algorithm is designed so that second order nonlinear transformations are initialized with the results of an affine registration, and successively higher order models are sequentially initialized with the best results obtained at lower orders.

Unlike intrasubject registration the brain images are edited to remove the scalp, skull, and dura when these structures are prominent (e.g., MRI data) before performing intersubject registration. The rationale for excluding these structures is that they are likely to vary in size and shape in ways that are distinct from the brain itself, making their inclusion likely to lessen the accuracy with which homologous structures within the brain are aligned. PET images of 
glucose metabolism or blood flow are not typically edited since the scalp, skull, and dura are not prominent.

For nonlinear models the usual AIR procedure (12) for removing biases associated with defining one image as the reference image and the other as the reslice image cannot be used because the nonlinear transformations are not analytically invertible. To compensate, the nonlinear algorithm includes in its cost function not only those voxels that are suprathreshold in the reference file but also those that are subthreshold in the reference file but that are suprathreshold in the corresponding voxel of the interpolated reslice image. Without this compensation, optimization of the cost function tends to enlarge the reslice image until it completely covers all the edges of the reference image.

Some default values for the nonlinear registration algorithm differ from those used by the linear algorithm. The primary termination criterion is based on the predicted change in the cost function (a value of 0.5 is the 8 bit default), and secondary termination criteria allow up to 50 total iterations at a given sampling density or 3 consecutive iterations without improvement in the cost function. To increase speed and compensate for the large number of derivatives that must be computed when the number of parameters is large, the final default sampling density is set at every ninth voxel. Routines are available for analytically combining nonlinear transformations with linear transformations to avoid the need to resample data more than once.

The benchmark work described here was performed on a Fujitsu HALstation 330 that is roughly two to three times faster than a Sun SPARCstation 10 when executing AIR registration algorithms.

\section{INTERSUBJECT REGISTRATION VALIDATION}

\section{Subjects and Data Acquisition}

A group of 22 healthy volunteers who had undergone PET scanning using $\mathrm{H}_{2}{ }^{15} \mathrm{O}$ and T1-weighted MR scanning at Hammersmith Hospital in London according to approved human subject protocols were identified. The subjects ranged in age from 20 to 51 years. Twenty were male and two were female. All but four were white. Those four were of West Indian, Japanese, Indian, and Middle Eastern descent. At least one subject was left- handed, and handedness data were not available for the other subjects. All subjects' MR images had already been manually edited to remove scalp, skull, and dura, and the images had already been manually transformed using trilinear interpolation and a rigid body model so that the midsagittal plane of the brain was centered and parallel to the primary image axes. The orientations of the brains in the sagittal plane were similar but not rigorously standardized. All images had already been interpolated to cubic voxels with isotropic voxel dimensions of $1.0 \mathrm{~mm}$. Proper positioning of the midsagittal plane was verified independently before inclusion in subsequent analysis.

The original MRI data sets had been acquired using a $1 \mathrm{~T}$ Picker HPQ Vista system with a T1-weighted radiofrequency

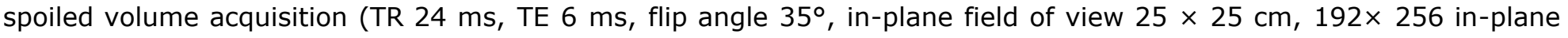
matrix with 128 secondary phase-encoding steps oversampled to 256 , resolution $1.3 \times 1.3 \times 1.5 \mathrm{~mm}$ ). The PET data had been acquired using a CTI 953B PET scanner (CTI, Knoxville, TN, U.S.A.) with septa removed for 3D acquisition. The scanner has an axial field of view of $10.65 \mathrm{~cm}$, and data were reconstructed to generate 31 axial images with a resolution of $8.5 \times 8.5 \times 4.3 \mathrm{~mm}$ full width at half-maximum. Subjects had performed a variety of functional tasks during acquisition of the(typically 12 ) $\mathrm{H}_{2}{ }^{15} \mathrm{O}$ scans. On average, subjects had received[almost equal to] $1,000 \mathrm{MBq}$ of $\mathrm{H}_{2}{ }^{15} \mathrm{O}$ per scan. The separate $\mathrm{H}_{2}{ }^{15} \mathrm{O}$ scans from each subject had been aligned to one another using an automated intramodality registration algorithm (13), and the resulting average image of each subject was made available for use here. All images were screened to verify image quality before inclusion in this study. MRI to PET registration for each subject was performed as described previously (14).

\section{Creation of Target Atlases}

Target atlases having the average size and shape of the brains of the 22 subjects were created for subsequent use in validation. The strategy for preparing the target atlases is illustrated in Fig. 1. First, all possible pairwise registrations of the 22 MRI data sets were performed using a 12 parameter affine spatial transformation model with the ratio image uniformity (RIU) cost function (12). This computationally intensive step was performed with a prototype implementation (15) of the AIR 3.0 registration algorithm and was not repeated with the newer algorithm. Aside from the quality and affine shape of the resulting target atlases, this particular use of the registration algorithm has no direct bearing on the 
subsequent validation studies performed using these target atlases. The matrix averaging and reconciliation procedures described in Appendix used the results of the pairwise registrations to define a transformation that would map each individual's MRI into a common space while preserving the average orientation, size, and affine shape of the group. Each MRI data set was resampled into this common space using trilinear interpolation, and voxel intensities were averaged across subjects to create an average brain MRI target atlas. With use of the known relationship between each MRI data set and the corresponding PET data set, all 22 PET data sets were also resampled into the same common space and similarly averaged to generate a co-registered average brain PET target atlas. Both target atlases had voxels that were $1.0 \mathrm{~mm}$ in each dimension.

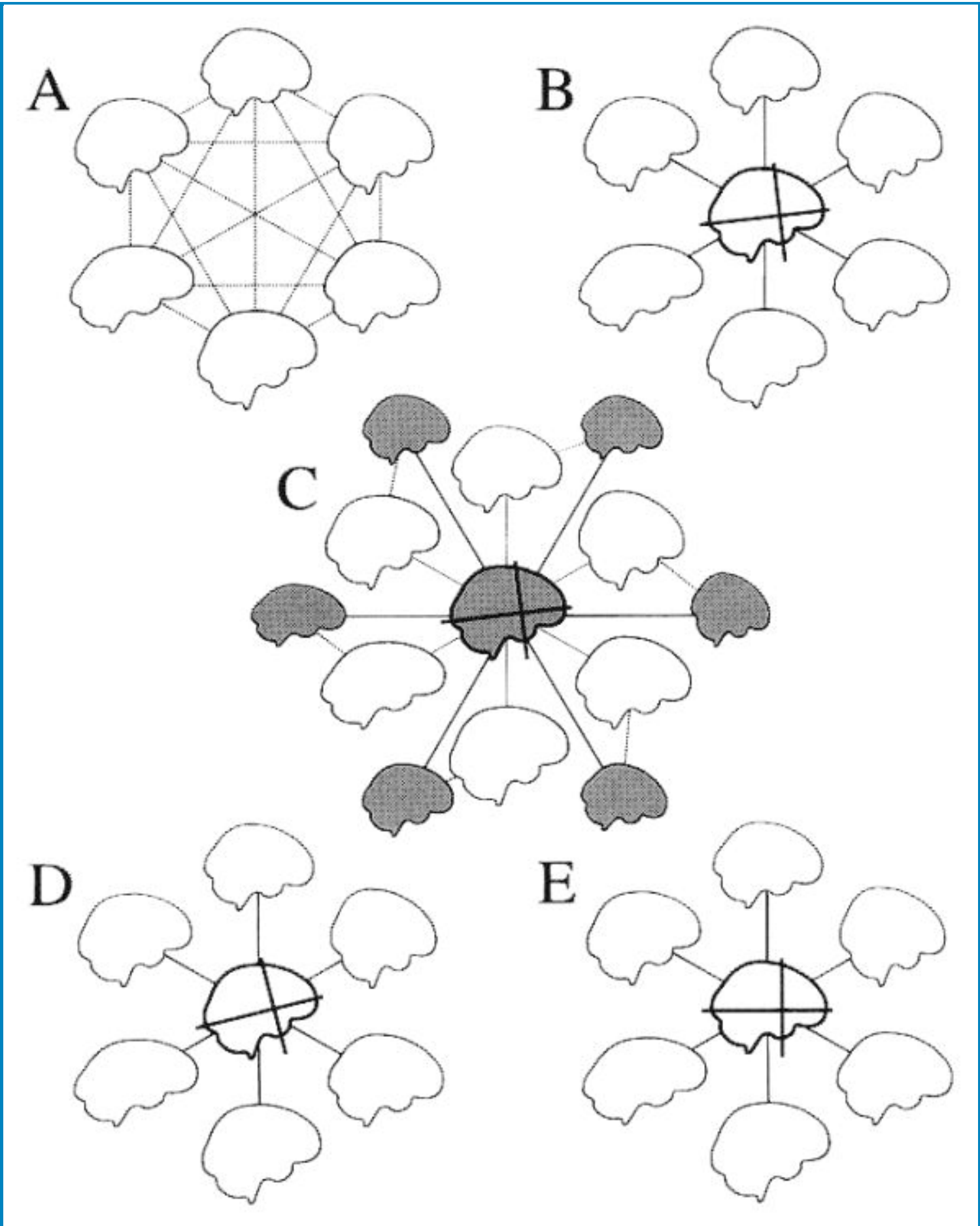

FIG. 1. Creation of target atlases (dark outlines). MR images are represented in white and PET images in gray. Dotted lines represent registration of the images connected by the line. Solid black lines represent resampling of data. The average orientation of the AC-PC line and of a line perpendicular to it passing through the AC are superimposed on the atlases. A: All possible pairwise registrations of the 22 MRI data sets were performed using an affine spatial 
transformation model. B: By the method described in Appendix, the results of the pairwise registrations were used to define affine transformations from each MR image into a space having the average size, shape, and orientation of the original images. All MR images were resampled into this space and averaged to create the MR average brain atlas. As illustrated, the resulting average AC-PC line was rotated $6.6^{\circ}$ from the horizontal. C: Each PET study was aligned to the corresponding MRI study. Combining this transformation (dashed lines) with the affine transformation used in (B) (gray lines) produced an affine transformation (black lines) used to resample the original PET data into an orientation, size, and shape identical to that of the average MRI atlas. The sampled PET images were averaged to create the average PET atlas.D: Combining the transformations used in (B) with an additional $7.5^{\circ}$ rotation around each of the three primary axes produced transformations that were used to resample and then average each MR scan to create a rotated average MRI atlas. E: Combining the transformations used in (B) with an additional $6.6^{\circ}$ rotation produced transformations that resulted in an atlas with the average position of the AC-PC line oriented horizontally.

Since all the MRI data sets had already been preprocessed to orient them with respect to the midsagittal plane, a second target atlas was specifically designed to test the ability of the algorithm to correctly identify oblique rotational misregistration of the data. This MRI target atlas was identical to the first MRI target atlas except that its relative position was rotated $7.5^{\circ}$ around the anteroposterior, left-right, and superoinferior axes (in that order). Since the exact relationship between the two target atlases was known, results of registrations using the rotated atlas could be mapped precisely back into the original unrotated atlas. The original PET data sets had not been preprocessed to correct for rotational misalignment, so a similar rotated PET atlas was not necessary.

It was also necessary to create a special version of the average brain MRI atlas with a properly oriented AC-PC line to evaluate and compare with the nine parameter Talairach model. The transformation from Talairach space to the average brain space described in the next section was used to define the additional rotation needed to create this atlas.

\section{Manual Registration}

To compare automated with manual registrations, the AC and PC were identified in each MR image anatomically, and the rigid body transformation necessary to horizontally align the superior margin of the AC with the inferior margin of the PC was performed. Since the images had already been rotated to make the midsagittal plane perpendicular to the leftright coordinate axis, this placed the images in the proper orientation for identifying the extreme edges of the cortex needed to map the images into Talairach space. Multiplicative factors were computed to rescale each brain independently along the $x, y$, and $z$ axes such that the bounding box dimensions of each rescaled brain image would equal the geometric mean of the corresponding bounding box dimension computed across all 22 brains. The two preceding transformations were mathematically combined into a single transformation, together with the necessary translations to ensure that the AC of each original image would be mapped to the same common coordinate location. This method is conceptually identical to that described by Lancaster et al. (3), except that the images were not rescaled to match the bounding box dimensions of the 1988 Talairach atlas (16). The final bounding box dimensions were as follows: left-right, $133 \mathrm{~mm}$; anteroposterior $174 \mathrm{~mm}$; and superoinferior $119 \mathrm{~mm}$. The corresponding dimensions in the 1988 Talairach atlas were 136, 172, and $118 \mathrm{~mm}$. The manual method just described will be referred to here as the manual Talairach method and the resulting space as Talairach space, but it should be noted that the method originally described by Talairach et al. actually used 129 parameter transformations joined together in a piecemeal fashion $(2,16)$ for a total of 13 independent parameters.

To define a transformation from Talairach space to a space that could be directly compared with the average brain MRI target atlas (which was not oriented on the AC-PC line), the matrix averaging procedure from Appendix was used to compute the average of the 22 manually derived transformations for mapping the MR images into Talairach space. Inversion of this transformation provided the average transformation from Talairach space back to the original images, thereby defining an average brain space analogous to the average brain MRI target space. The resulting transformation from Talairach space to the average brain space turned out to be a rigid body transformation (to three significant figures) and specified a rotation of $6.6^{\circ}$ around the right-left axis.

\section{Validation Using Landmarks}

To define landmarks for anatomic validation, each of the 22 original brain data sets was rendered three dimensionally, and selected landmarks were traced manually by one of us using anatomic criteria. The landmarks chosen were the right and left precental and postcentral gyri, the right and left parietooccipital and calcarine sulci, and the right 
and left superotemporal gyri including the posterior extension of this gyrus into the parietal lobe. Gyri were traced along their crests at the gray-white interface. The sulci, which were located on the mesial surface of the brain, were traced along their centers as viewed mesially. Consequently, the portion of the sulcus closest to the midline, not the depth of the sulcus, determined the location. The landmarks were traced before reviewing the results of any registration, and the same landmark tracings were used to evaluate all of the intersubject registrations. This was accomplished by mathematically projecting each traced landmark point on the 3D renderings back into the original 3D volume of data and then onward into the final common space using the particular set of registration parameters being evaluated. For nonlinear transformation models, this required numerical inversion of the spatial transformation at each voxel using an iterative multivariate calculus-based search strategy. This approach ensures that any inaccuracies in landmark identification are unbiased with respect to the model or method being tested and equally likely to be detrimental to the apparent performance of any of the registration strategies.

The manually identified AC and PC were also used as anatomic landmarks. The 3D coordinates of these two landmarks in the original images were projected into the average brain atlas space using the transformation being evaluated. To determine the correct location of the AC and the correct slope of the AC-PC line in the atlas, the two commissural landmarks were also projected into the average brain MRI atlas using the same transformations originally used to create the atlas. The resulting mean coordinate location of the AC was defined as the origin of the atlas. For each registration method evaluated, the mean coordinate location of the AC was determined, and the mean and maximal 3D distances of each individual's AC from this mean coordinate location were computed. The mean angulation of the AC-PC line relative to the horizontal axis in sagittal projection was also determined, and the mean and maximum angular deviations from this mean angulation were established for the 22 individual AC-PC lines in the atlas space. Each of the individual MR images was registered to the average brain MRI atlas using the 7 and 12 parameter spatial transformation models with the RIU and scaled least squared (SLS) difference cost functions. Similarly, the nine parameter model was used to align each MRI to the special average brain MRI atlas in Talairach space with these cost functions. The average brain MRI atlas that had been rotated by $7.5^{\circ}$ around each axis was used as a target for a separate set of registrations using the 12 parameter spatial transformation model. For these registrations, the maximum number of iterations allowed at a given sampling interval was increased from the linear registration algorithm default of 25 to a value of 50 after noting that the cost function was often still decreasing after 25 iterations. The original PET data sets (not the resampled versions that had been individually aligned to the MRI scans) were registered to the average brain PET atlas using the 12 parameter model. For the RIU cost function, a convergence threshold of $10^{-5}$ was used, and for the SLS cost function, a convergence threshold of 0.1 . MRI registrations initially sampled every 81st voxel. PET registrations initially sampled every 27 th voxel. The final sampling interval included every voxel for both types of data. The cost function was computed in an unbiased manner by inverting the role of reference image and reslice image to recompute the cost function with each iteration.

Nonlinear registrations used the average brain MRI atlas for MRI data and the average brain PET atlas for PET data. Full Newton type minimization and Marquardt-like minimization (12) were both evaluated. The latter method is designated here by appending "LM" to the cost function. Second order nonlinear registrations were initialized with the results previously obtained with the linear 12 parameter model using the SLS cost function. Higher order nonlinear models were initialized with the results obtained from the next lower order nonlinear model that used the same minimization strategy. A convergence threshold of 0.5 was used. Initial sampling of data was every 81st voxel for MRI data and every 27 th voxel for PET data. Final sampling was at every ninth voxel for both data types. Up to 50 iterations were allowed at each sampling density. For MRI data, up to three consecutive iterations without improvement in the cost function were allowed before increasing the sampling density or terminating. For PET data the sampling interval was immediately decreased or the algorithm terminated if the cost function was not improved on any iteration.

Each spatial transformation model and method was assessed by using the resulting transformations to map the traced landmarks from all 22 subjects into a single volume in the space defined by the average brain MRI atlas so that the overlap of homologous landmarks could be evaluated both visually and quantitatively. Since the cortical landmarks consisted of sets of voxel locations defining homologous structures, rather than of individual point landmarks having oneto-one correspondences, quantitative comparisons could not be based on errors in the locations of individual homologous points. Instead, for a given landmark voxel in one subject, the distances to each of the voxels comprising the corresponding landmark in a second subject were calculated, and the shortest of these distances was defined as the 
distance function for that landmark voxel with respect to that second subject. This resulted in 21 different distance functions (one for each of the other subjects) for each landmark voxel of a given subject. These 21 distance functions were then averaged to produce a mean distance function for that particular landmark voxel in that particular subject. Over 90,000 mean distance functions were computed for each registration strategy, corresponding to several hundred voxels per cortical landmark for each of five landmarks in both hemispheres of 22 subjects. The cumulative distributions of these mean distance functions for each registration strategy were then displayed graphically using S-plus (MathSoft, Seattle, WA, U.S.A.).

\section{RESULTS}

Table 1 shows the specific intersubject MRI registrations that were performed with the SLS cost function and lists the average registration times. Data regarding the location of the AC and the angulation of the AC-PC line are also shown. The average error in the angulation of the AC-PC line was no more than $0.3^{\circ}$ and the standard deviation of the angulation no more than $1.5^{\circ}$ for any registration strategy. Errors in the location of the AC decreased as the number of parameters in the transformation model was increased from 7 to 60 and remained stable for fourth and fifth order nonlinear transformations. Excluding the seven parameter model, the mean 3D error in the location of the AC was no more than $1.3 \mathrm{~mm}$ with a standard deviation of no more than $0.6 \mathrm{~mm}$.

\begin{tabular}{|c|c|c|c|c|c|c|c|}
\hline Target & $\begin{array}{c}\text { Model } \\
\text { parameters }\end{array}$ & $\begin{array}{c}\text { Cost } \\
\text { function }\end{array}$ & $\begin{array}{l}\text { Canvergence } \\
\text { threshold }\end{array}$ & $\begin{array}{c}\text { Final } \\
\text { sampling }\end{array}$ & $\begin{array}{c}\text { Mean } \\
\text { registration } \\
\text { time (min) }\end{array}$ & $\begin{array}{l}\text { Mean error } \\
\pm \mathrm{SD}(\mathrm{mm}) \\
\text { in location } \\
\text { of anterior } \\
\text { comanissure }\end{array}$ & $\begin{array}{l}\text { Mean error } \\
=\mathrm{SD} \text { (degrees) } \\
\text { in angulation } \\
\text { of } \mathrm{AC}-\mathrm{PC} \text { line }\end{array}$ \\
\hline Pairwise MRI's & 12 & RIU & & 1 & & $1.1 \pm 0.5$ & $0 \pm 1.3$ \\
\hline Talairach space & 9 & manual & & & & $0 \pm 0$ & $0 \pm 0$ \\
\hline MRI atlas & 7 & SLS & 0.1 & 1 & 7.1 & $1.4 \pm 0.8$ & $0.2 \pm 1.3$ \\
\hline \multicolumn{8}{|l|}{ MRI atlas in } \\
\hline Talairach space & 9 & SLS & 0.1 & 1 & 12.2 & $1.3 \pm 0.6$ & $0.3 \pm 1.5$ \\
\hline MRI atlas & 12 & SLS & 0.1 & $i$ & 8.2 & $1.2 \pm 0.6$ & $0.2 \pm 1.2$ \\
\hline Rotated MRl atlas & 12 & SLS & 0.1 & 1 & 8.8 & $1.2 \pm 0.6$ & $0.0 \pm 1.3$ \\
\hline MRI atlas & 30 & SLS & 0.5 & 9 & 11.6 & $1.1 \pm 0.5$ & $0.2 \pm 1.2$ \\
\hline MRI atlas & 60 & SLS & 0.5 & 9 & 23.6 & $1.0 \pm 0.4$ & $0.1 \pm 1.1$ \\
\hline MRI atlas & 105 & SLS & 0.5 & 9 & 62.2 & $1.1 \pm 0.4$ & $0.1 \pm 1.2$ \\
\hline MRI atlas & 168 & SLS & 0.5 & 9 & 167 & $1.1 \pm 0.6$ & $0.1 \pm 1.3$ \\
\hline MRI attas & 30 & SLS-LM & 0.5 & 9 & 10.2 & $1.1 \pm 0.5$ & $0.2 \pm 1.2$ \\
\hline MRI atlas & 60 & SLS-LM & 0.5 & 9 & 15.5 & $1.0 \pm 0.5$ & $0.1 \pm 1.2$ \\
\hline MRI atlas & 105 & SLS-LM & 0.5 & 9 & 30.6 & $1.1 \pm 0.5$ & $0.1 \pm 1.1$ \\
\hline MRI atlas & 168 & SLS-LM & 0.5 & 9 & 82 & $1.1 \pm 0.6$ & $0.2 \pm 1.4$ \\
\hline \multicolumn{8}{|c|}{ 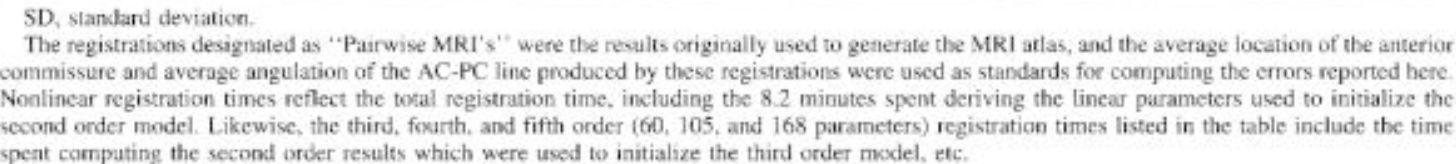 } \\
\hline
\end{tabular}

TABLE 1. Intersubject registration speed and accuracy in identifying the anterior commissure and angulation of the AC$P C$ line in MRI data as a function of registration method

Table 2 shows corresponding values for PET registrations using affine or nonlinear models with the SLS cost function. In using the SLS cost function to align PET data with the affine model, there was one registration failure due to a nonpositive definite Hessian matrix(indicating a nearby local maximum or saddle point that disrupts minimization) during the initial iteration. Satisfactory results were obtained in all cases with the RIU cost function. Inspection of the one problematic PET data set revealed that its intensity was substantially lower than that of all the other PET images. Changing the default initial intensity scaling factor by a factor of 2 for that particular registration rectified the problem, and the resulting registration was used for the subsequent analysis reported here. Analysis of the individual transformations showed that the PET images required rotation by as much as $23^{\circ}$ (mostly in the sagittal plane) to match the average PET atlas. Eighteen of the 22 PET images required rotations between 5 and $15^{\circ}$. After registration, the average error in the angulation of the AC-PC line was no more than $0.2^{\circ}$ with a standard deviation of no more than $1.8^{\circ}$ for any strategy investigated. Mean errors in the location of the AC were no more than $1.5 \mathrm{~mm}$ with a standard deviation of no more than $0.8 \mathrm{~mm}$. Values are not listed for the SLS cost function with full Newton type minimization for third, fourth, or fifth order nonlinear models because these models consistently resulted in problems with saddle points or local maxima when extended to higher orders with PET data, preventing further minimization. With Marquardt-like minimization (SLS-LM), it was possible to optimize these registrations at higher orders, but the algorithm did not always 
find new higher order parameters that actually improved the cost function. After fifth order registration, only 1 of the 22 registrations actually contained any fifth order terms. Eleven included only terms up to fourth order, nine only to third order, and one only to second order.

\begin{tabular}{|c|c|c|c|c|c|c|c|}
\hline Target & $\begin{array}{l}\text { Model } \\
\text { parameters }\end{array}$ & $\begin{array}{c}\text { Cost } \\
\text { function }\end{array}$ & $\begin{array}{l}\text { Converyence } \\
\text { threstiold }\end{array}$ & $\begin{array}{c}\text { Funal } \\
\text { sampling }\end{array}$ & $\begin{array}{c}\text { Mean } \\
\text { registration } \\
\text { time (min) }\end{array}$ & $\begin{array}{l}\text { Mean errox } \\
\pm \mathrm{SD} \text { (mm) } \\
\text { in location } \\
\text { of anterior } \\
\text { commissure }\end{array}$ & $\begin{array}{l}\text { Mean efror } \\
\pm \text { SD (degrces) } \\
\text { in angulation } \\
\text { of } \mathrm{AC} \text { - PC line }\end{array}$ \\
\hline PET atlas & 12 & SLS & 0.1 & 1 & 3.7 & $1.4 \pm 0.8$ & $0.1=1.7$ \\
\hline PET atlas & 30 & SLS & 0.5 & 9 & 5.2 & $1.5 \pm 0.5$ & $0.1 \pm 1.8$ \\
\hline PET atias & 60 & SIS & 0.5 & 9 & 9.4 & $1.4 \pm 0.5$ & $0.1 \neq 1.5$ \\
\hline PET atias & 30 & SLS-LM & 0.5 & 9 & 4.5 & $1.4 \pm 0.6$ & $0.2 \pm 1.8$ \\
\hline PET allas & 60 & SLS-LM & 0.5 & 9 & 6.4 & $1.4 \pm 0.5$ & $0.1 \pm 1.4$ \\
\hline PET arlas & 105 & SLS-LM & 0.5 & y & 10.9 & $1.4 \pm 0.5$ & $0.2 \pm 1.5$ \\
\hline PET atlas & 168 & SLS-LM & 0.5 & 9 & 23.6 & $1.4 \pm 0.5$ & $0.2 \pm 1.5$ \\
\hline
\end{tabular}

TABLE 2. Intersubject registration speed and accuracy in identifying the anterior commissure and angulation of the AC$P C$ line in PET data as a function of registration method

The results of linear registrations with the RIU cost function were very similar to those obtained with the SLS cost function, though registration times were always longer with the RIU cost function. Consequently, in the interest of space, the details of the RIU registrations are not reported or shown here. Figure 2 shows the superimposition of homologous landmarks achieved with specific linear registration strategies, and Fig. 3 shows similar results for affine and nonlinear registration strategies. Except the seven parameter global rescaling model, all automated strategies produced results superior to those obtained using manual Talairach registration. The typical locations of the landmarks obtained with Talairach registration were extremely similar to their typical locations obtained with the automated methods. Increasing the number of parameters in the model led to subtle but noticeable improvements from one model to the next for MRI data. For the PET data shown in Fig. 3, the second order nonlinear model was better than the affine model, but additional benefit with higher order models is difficult to discern. Figures 2 and 3 also serve to verify that the quantitative values shown in Fig. 4 are not artifacts caused by systematic differences in overall scaling of the images by different models. Figure 4 compares the cortical landmark results using the cumulative distributions of the mean distance function as a metric, confirming the qualitative results already described. Direct comparison of cumulative distributions obtained for the MRI data sets with full Newton type minimization versus Marquardt-like minimization invariably revealed almost identical distributions (not shown). 


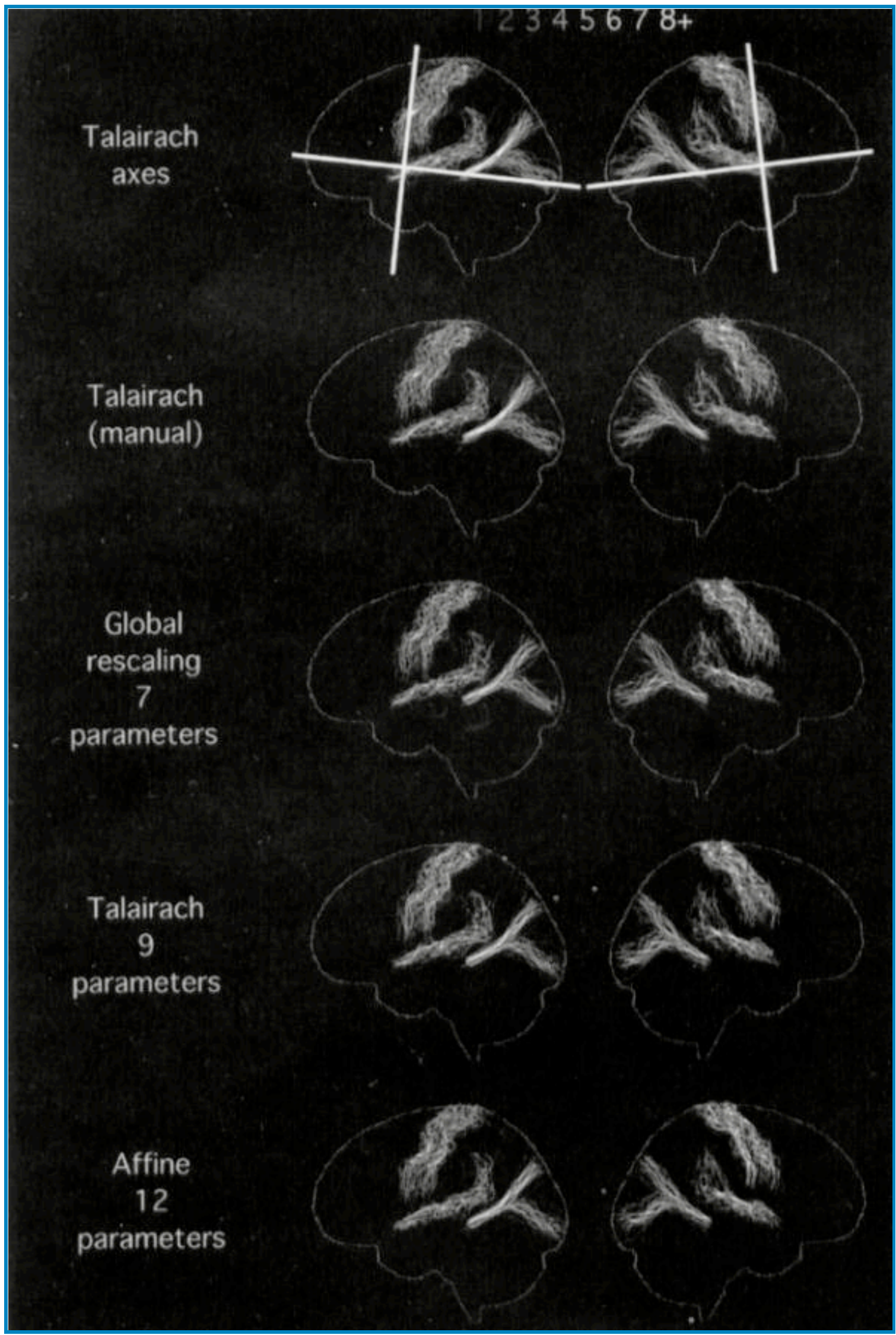

FIG. 2. Comparison of manual and automated linear registration methods for MRI data. The images show superimposition of cortical landmarks in sagittal projection in the space defined by the average brain target atlas. The Talairach axes are shown in the first row. Left hemisphere landmarks are shown on the left and right hemisphere landmarks on the right. Precentral, postcentral, and superotemporal gyri are shown along with the parietooccipital and calcarine sulci. The intensity scale indicates the number of subjects with homologous landmarks at a given voxel location. In areas where two different landmarks overlap, the scaling reflects the landmark with the greatest homologous overlap. 
The landmarks in the first and second rows were aligned manually using a nine parameter Talairach method. Registrations for the remaining rows were automated and used the SLS cost function. The average brain MRI atlas was used as the target for the seven parameter model. The average brain MRI atlas in Talairach space was used as the target for the 9 parameter model, and the rotated average brain MRI atlas was used as the target for the 12 parameter model.

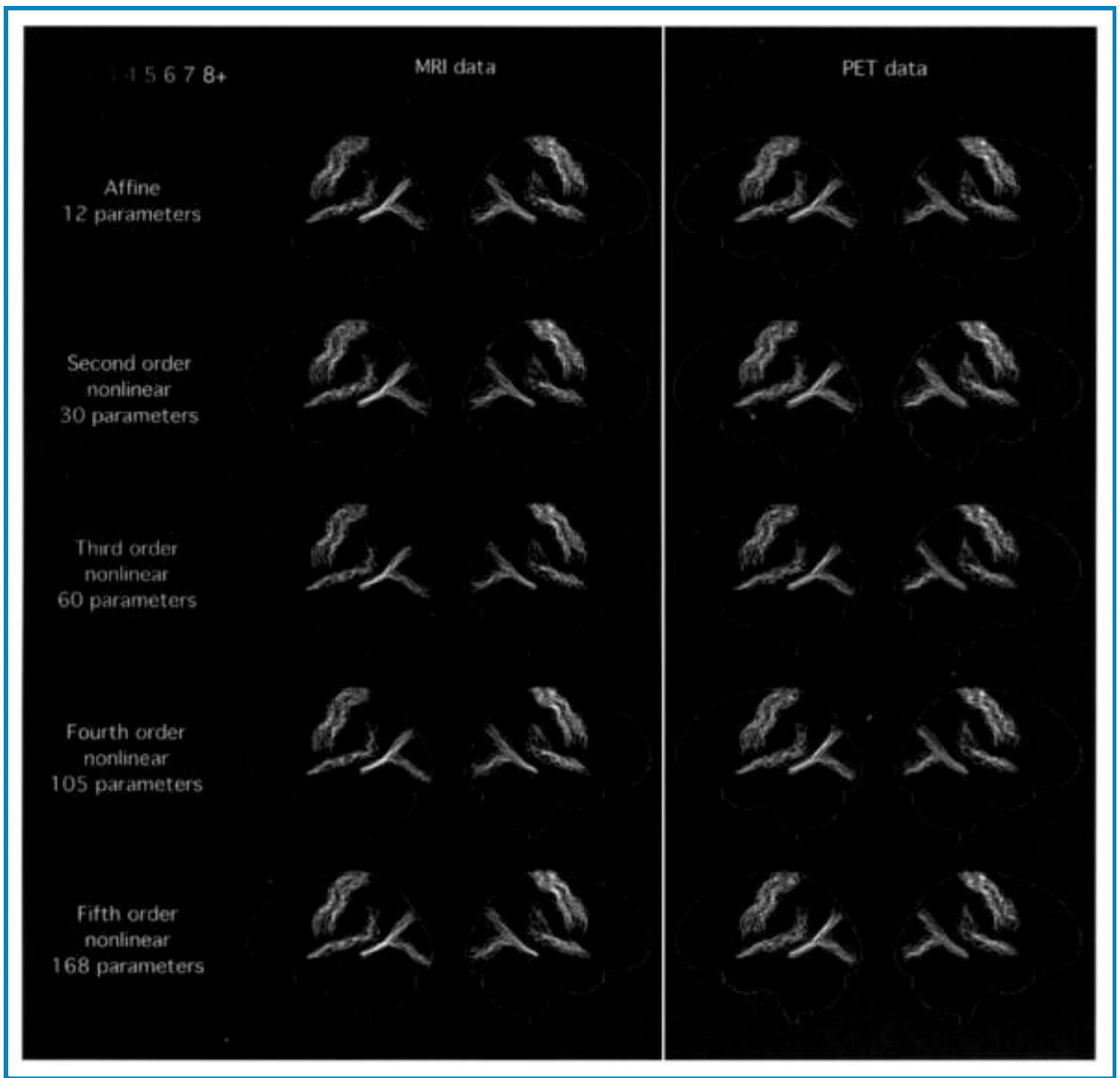

FIG. 3. Comparison of linear and nonlinear automated registration for intersubject registration of MRI data sets and PET data sets. Landmarks are illustrated as in Fig. 2. The average brain MRI atlas was used as the target for all MRI registrations and the average brain PET atlas for all PET registrations. For MRI registrations, the SLS cost function was used. PET registrations were based on the SLS-LM cost function. 


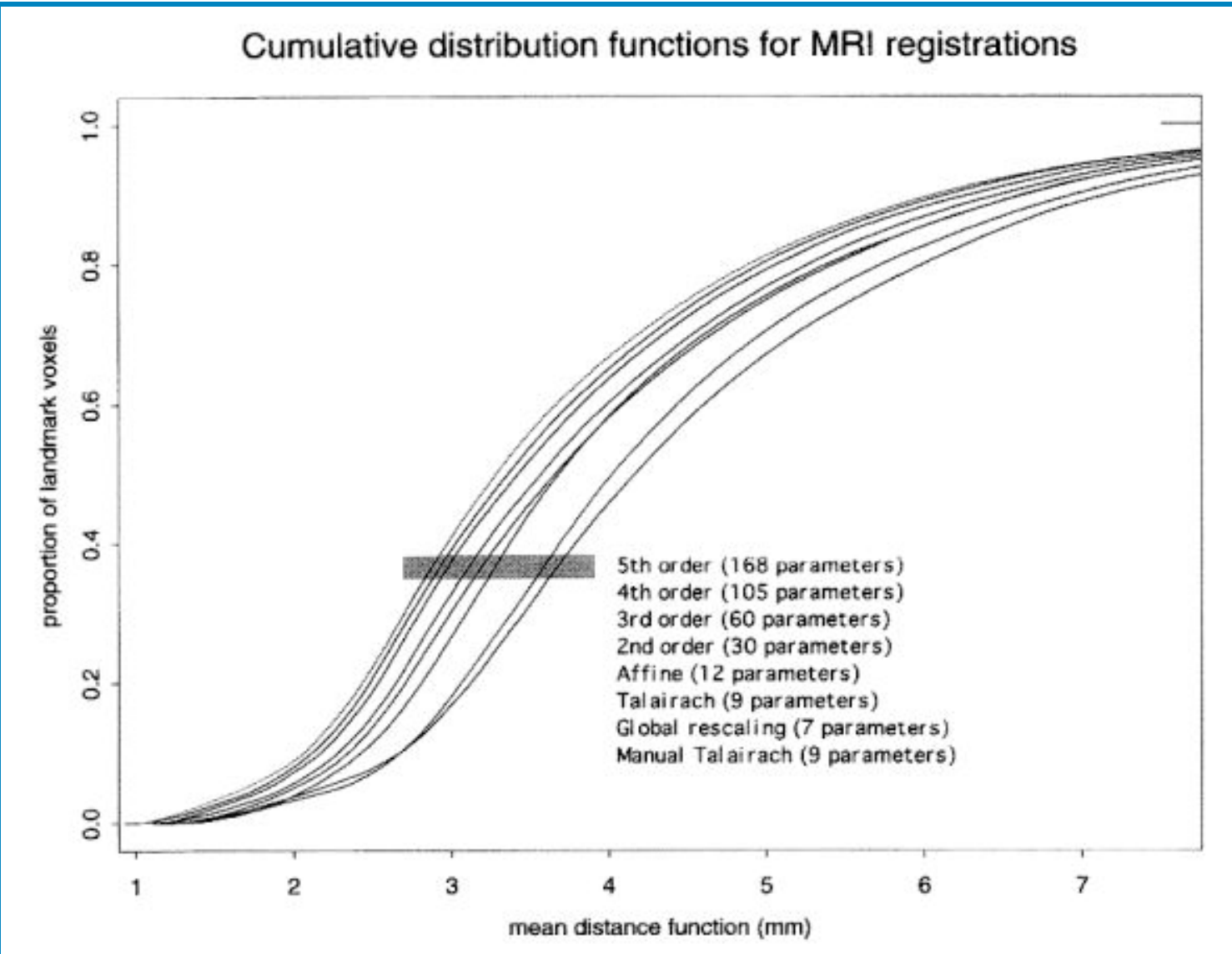

Cumulative distribution functions for PET registrations

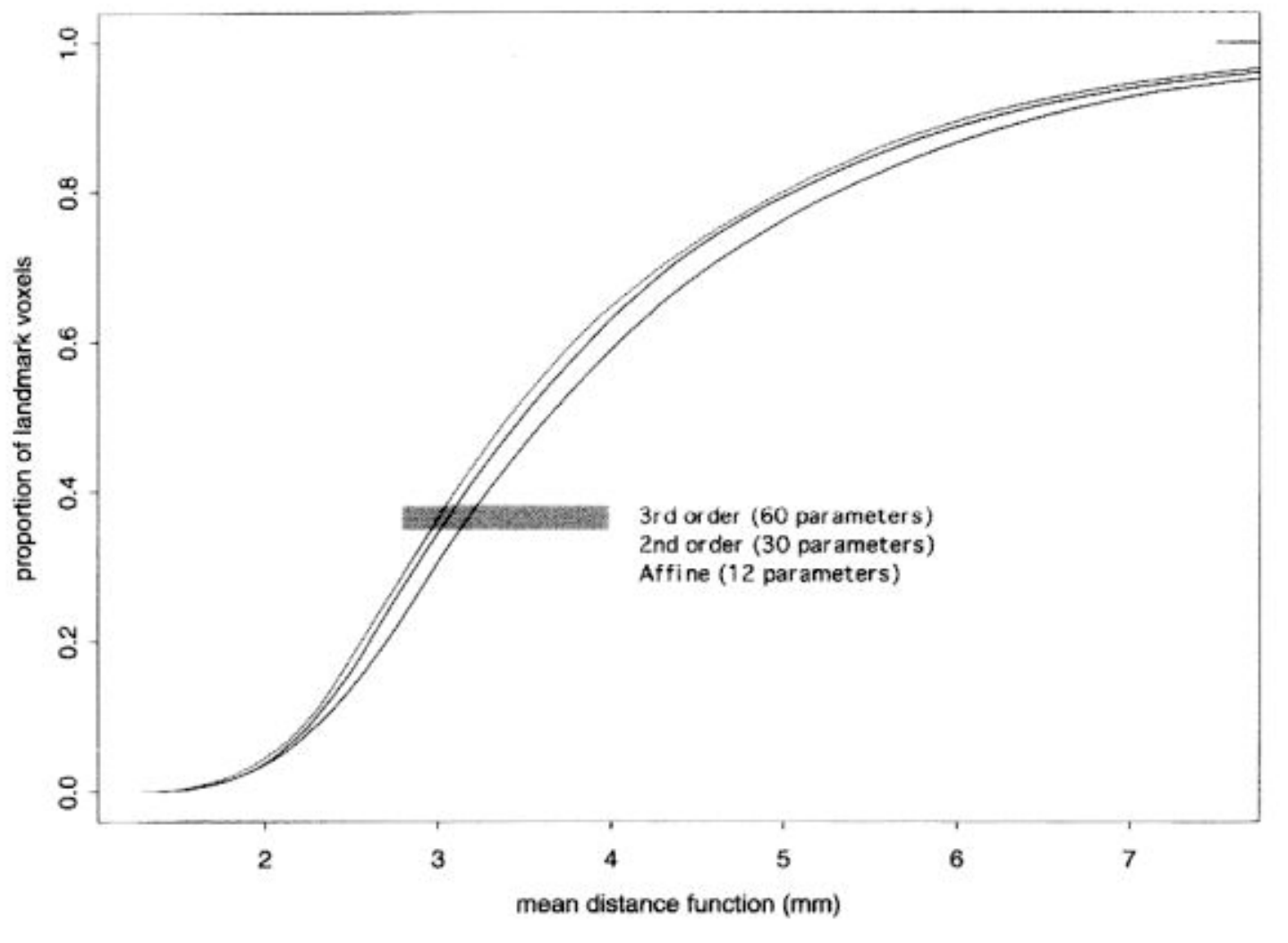

FIG. 4. Quantitative comparisons of different spatial transformation models for MRI (top) and PET (bottom) data. The distance function reflects the shortest distance from a given point to a homologous landmark from another subject, and the mean distance function averages this value across all other subjects. For a given value of the mean distance function on the $x$ axis, the proportion of landmark voxels having a mean distance function less than or equal to that value is plotted on the $y$ axis. Each registration method is represented by a separate curve. Curves that are shifted to the left 
reflect better superimposition of homologous landmarks than the curves to their right. The gray bars are included solely for labeling purposes. The registration methods are listed in the order in which they intersect the gray bar, proceeding from left to right.

Figures 5 and 6 show 3D renderings of individuals chosen because of their dissimilarity to one another after manual Talairach registration. Images created using successively higher order spatial transformation models are illustrated. The figures show that the transformations alter the 3D shape and size of the brain but not the basic underlying sulcal and gyral patterns.

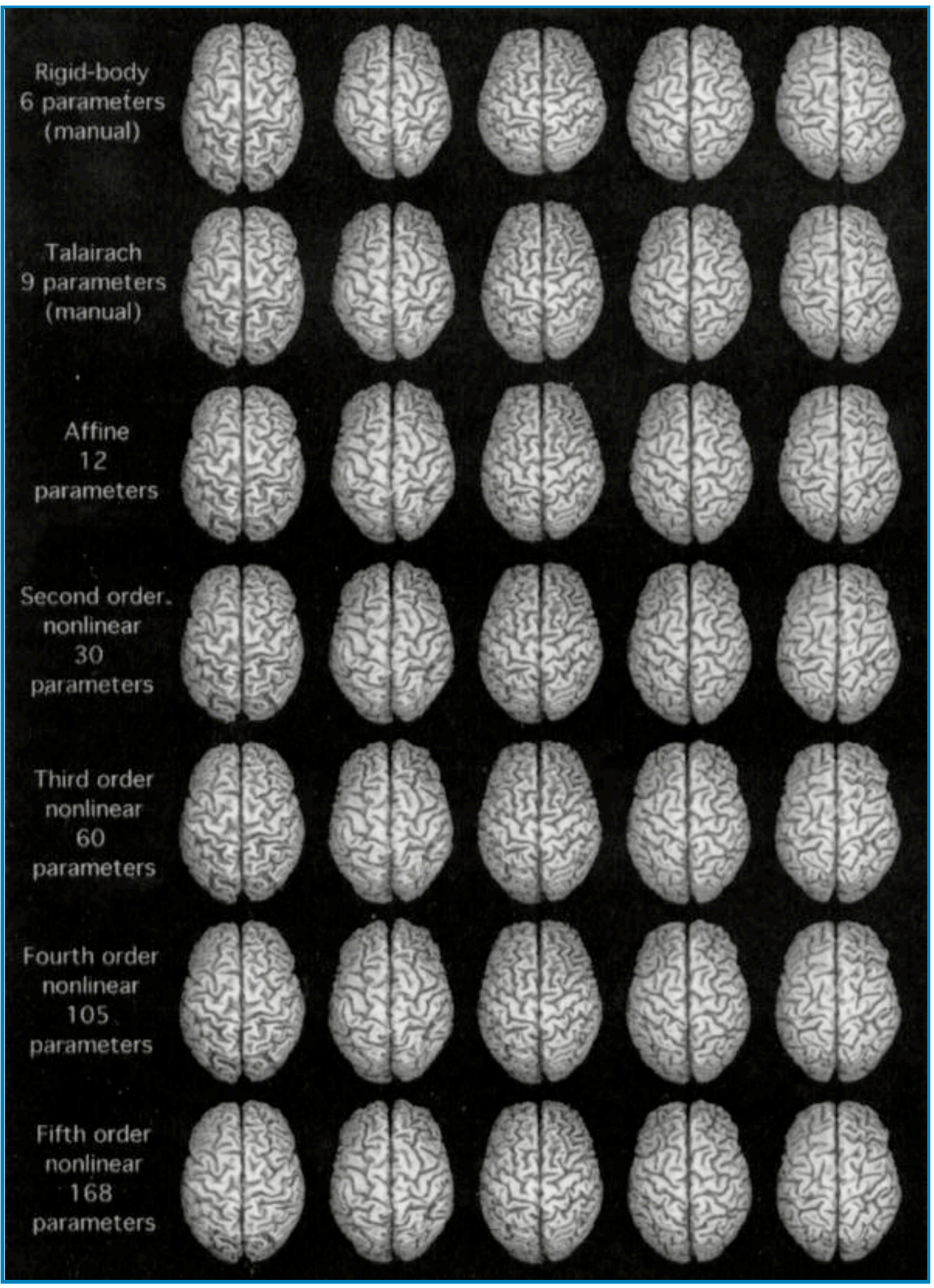


FIG. 5. Surface renderings of the brains of five subjects selected based on their dissimilarity after manual Talairach registration. The images are rendered from above at an average angle of $6.6^{\circ}$ to the AC-PC line. Note the gradual increase in similarity of brain shape as the number of parameters in the spatial transformation model is increased. All models retain the overall pattern of sulci and gyri evident in the original undistorted images.

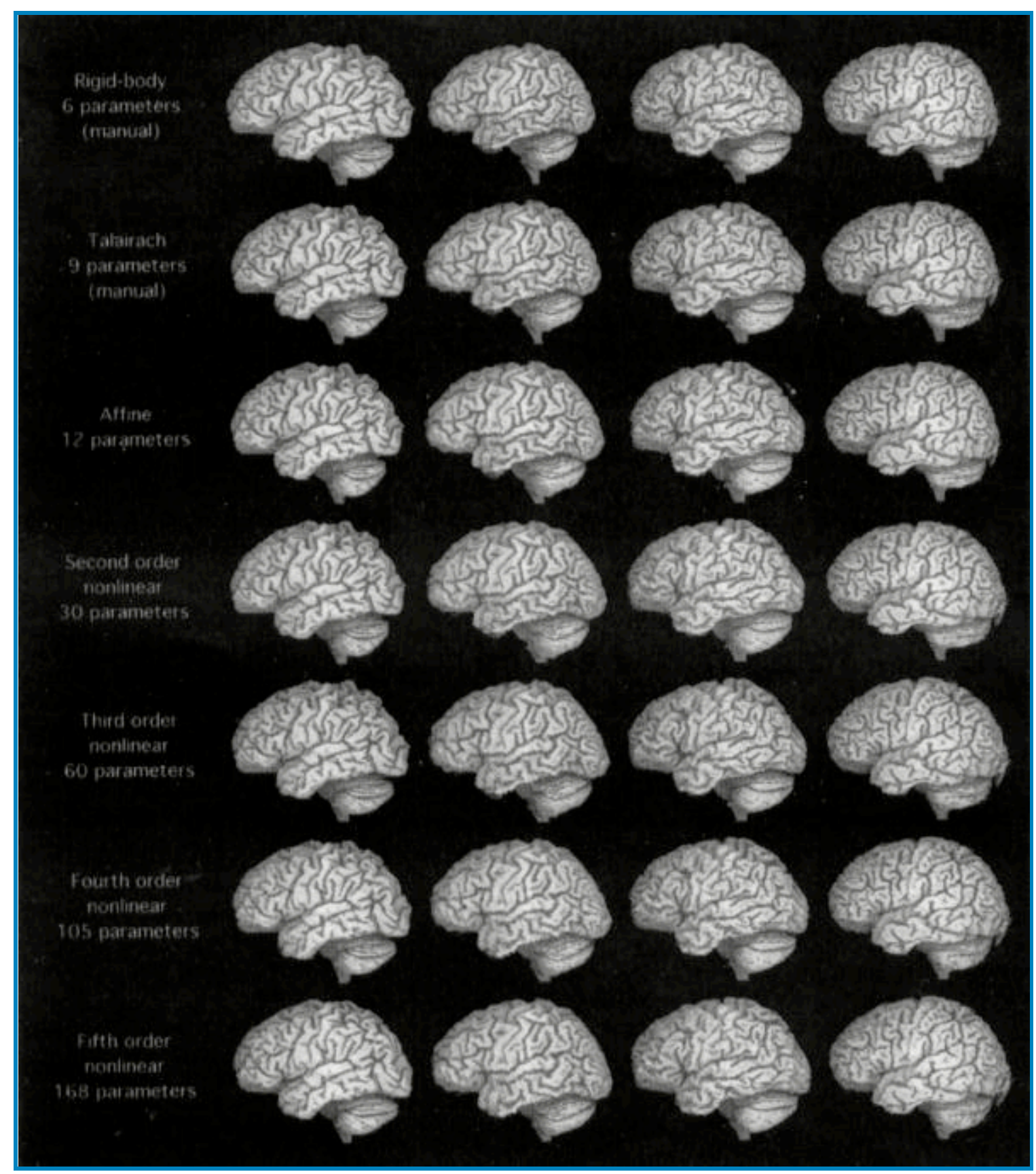

FIG. 6. Surface renderings of the left side of the brain of four subjects selected based on their dissimilarity after manual Talairach registration. The images are positioned with the AC-PC line oriented at an average angle of $6.6^{\circ}$. Note the gradual increase in similarity of brain shape as the number of parameters in the spatial transformation model is increased and the preservation of the overall pattern of sulci and gyri.

\section{DISCUSSION}

The linear and nonlinear registration algorithms described here provide reliable methods for aligning images from a group of subjects to allow intersubject comparisons or to allow derivation of standardized coordinate locations. For MR images, editing of the scalp, skull, and dura is required. Automated registration of MRI or PET data using these algorithms consistently resulted in better superimposition of homologous cortical landmarks than nine parameter manual registration based on the Talairach landmarks as identified on MRI. The AC and the orientation of the AC-PC line were also aligned quite accurately using the automated approach. For linear registration, only one registration failure occurred 
as a result of a poorly scaled image, a problem that was easily corrected by initializing the algorithm with a more appropriate intensity scaling factor. For nonlinear registration of MRI data, there were no registration failures. Nonlinear registration of PET data was not invariably successful in identifying high order nonlinear corrections but did always identify lower order nonlinear corrections that produced better results than linear registration.

\section{Spatial Transformation Model}

Biological considerations place very few constraints on the choice of spatial transformation model for inter- subject registration. Choosing the most general spatial transformation model available is not necessarily the most appropriate strategy. For example, linear affine transformations have unique advantages over nonlinear transformations of being analytically invertible, of maintaining uniform spatial resolution, and of being compatible with the most efficient image resampling methods. More nonlinear parameters do not necessarily guarantee better results, and any empirically demonstrated improvements in accuracy must be weighed against associated increases in computational burden.

For linear registration, the validation studies show that the seven parameter model is a little faster than the affine model but is inferior in terms of accuracy. The affine model is no slower than the nine parameter Talairach model and achieves better superimposition of homologous landmarks. It also avoids a unique disadvantage of the nine parameter Talairach model, which requires specific orientation of the target before registration.

Nonlinear models lead to further improvements in the match of the shape of the brain to that of the target and in the superimposition of homologous cortical landmarks. For PET data, fourth and fifth order polynomial models provide no additional benefit over a third order model and are considerably slower. For MRI data, continued improvement is seen through the fifth order polynomial model at the expense of increasingly large computation times.

Nonlinear polynomial spatial transformation models can potentially produce distortions that disrupt the expected oneto-one correspondence between the original and the resampled image. For example, a portion of the brain in the original image could be represented twice in the resampled image. Despite the absence of any absolute formalism to prevent such reduplications, none were encountered here within the brain itself. Reduplications of data outside the brain could sometimes be demonstrated by replacing the edited brain image with its unedited counterpart before resampling. Reduplications are penalized by the cost function, so the field of view of the target space should be kept as small as possible when using nonlinear polynomial models.

Except for the nine parameter Talairach model, all of the spatial transformation models have the property of invariance under affine transformation. Consequently, the specific orientation of the brain within the images has no effect on the constraints imposed by the spatial transformation model. Spatial invariance also makes it easy to mathematically redefine the target space. If it is decided after registration that the target actually should have been rotated by $10^{\circ}$, it is not necessary to reregister the images to be able to resample the data to match this new target orientation.

\section{Optimizing Registration Speed}

Based on the successful use of sparse sampling as an effective means of increasing speed with minimal loss of accuracy for intrasubject registration (12), sparse sampling was used here routinely for nonlinear intersubject registration. The continued improvement in registration accuracy when proceeding from linear models with full sampling to nonlinear models with sparse sampling provides empiric support for this approach. The RIU cost function was slower and no more accurate than the SLS cost function for intersubject registration with linear models and was therefore not evaluated for nonlinear models. Marquardt-like minimization was faster, more robust, and at least as accurate as full Newton type minimization with the SLS cost function.

\section{Evaluating Intersubject Registration Accuracy}

The lack of gold standard transformations makes evaluation of intersubject registration accuracy difficult. For nonlinear spatial transformation models, it is especially important that the assessment of accuracy not rely solely on simple visual inspection of resampled images such as those shown in Figs. 5 and 6 . High order nonlinear transformations may be able to create and destroy sulci and gyri to make the resampled image look similar to the target. The resulting images may suggest excellent registration, and only discrepancies in the locations of homologous anatomic landmarks identified in the original images and mapped into the target atlas will reveal whether the procedure has failed to achieve 
its true objective. The results in Figs. 2-4 show that the relatively low order nonlinear transformations used here do indeed lead to genuine improvements in the mapping of homologous anatomic landmarks to consistent locations.

\section{Registration Targets and Standardized Coordinates}

For the validation work described here, the target atlases were created by averaging images of all of the subjects after preliminary affine registration. Additional analyses not reported here show that qualitatively similar overlap of homologous landmarks can be achieved by targeting data of a single subject or by targeting averaged data after preliminary fifth order nonlinear registration. However, targets with different shapes do result in different typical coordinates for landmarks, even when the final target orientation has been standardized using the Talairach rules. The simplest way to standardize target shape is to generate the target by averaging brain images after preliminary registration using the manual Talairach method $(5,17)$. Alternatively, the matrix averaging technique described in Appendix, which generalizes the Talairach rescaling procedure to apply to affine transformations, can be used to define the target shape from a preliminary set of affine registrations to a single arbitrarily chosen target or from a preliminary set of all possible pairwise affine registrations. Ideally, the Talairach procedure should be further generalized to allow a definition of the average brain shape based on nonlinear registrations so that the quality of the target atlas can be improved.

In routine use, the images used to create the registration target will probably not be the same images that are being registered to the target. Consistent use of a single, well designed target atlas may lead to better reproducibility of coordinate locations than creation of a new atlas de novo for every experiment. The accuracy of the methods described here when the target atlas is derived using different subjects and less standardized imaging parameters warrants further investigation.

\section{Generality of Results}

Although designed for intersubject registration of 3D human brain data, the AIR intersubject registration method may be useful in other contexts as well. Since the method does not rely on explicit landmark identification, it should be applicable to brain images from other species and possibly to images of other parts of the body. The nonlinear registration tools may also be helpful for intrasubject registration of developmental data and for data containing nonlinear image distortions. Two-dimensional implementations of the method may be helpful in correcting distortions in 3D photographic images. Finally, the transformations derived by the nonlinear algorithm may prove useful in characterizing and measuring low order morphological differences, and removal of these low order differences may reveal higher order distinctions that are relevant to human behavior and to diseases that affect the brain and its morphology.

Acknowledgment: We thank Dr. Gereon Fink for making available some of the MRI and PET data sets. This work was supported by a grant from the Charles A. Dana Foundation, by National Institute of Neurological Disorders and Stroke grant 1 K08 NS-01646, Department of Energy contract DE-FCO3-87ER60615, and generous gifts from the Pierson-Lovelace Foundation, the Ahmanson Foundation, the Tamkin Foundation, the North Star Fund, and the Brain Mapping Medical Research Organization.

\section{REFERENCES}

1. Rademacher J, Werner C, Morosan P, Schleicher A, Zilles K, Steinmetz H. Localization and variability of cytoarchitectonic areas in the human superior temporal cortex. Neuroimage 1996;3:S456. [Context Link]

2. Talairach J, Szikla G, Tournoux P, et al. Atlas d'anatomie stéréotaxique du télencéphale. Paris: Masson, 1967. [Context Link]

3. Lancaster JL, Glass TG, Lankipalli BR, Downs H, Mayberg H, Fox PT. A modality-independent approach to spatial normalization of tomographic images of the human brain. Hum Brain Map 1995;3:209-23. [Context Link]

4. Kosugi $Y$, Sase M, Kuwatani $\mathrm{H}$, et al. Neural network mapping for nonlinear stereotactic normalization of brain MR images. $J$ Comput Assist Tomogr 1993;17:455-60. [Context Link]

5. Friston KJ, Frith CD, Liddle PF, Frackowiak RS. Plastic transformation of PET images. J Comput Assist Tomogr 1991;15:634-9. Bibliographic Links | [Context Link]

6. Collins DL, Holmes CJ, Peters TM, Evans AC. Automatic 3-D model-based neuroanatomical segmentation. Hum Brain Map 1995;3:190-208. [Context Link]

7. Haller JW, Christensen GE, Joshi SC, et al. Hippocampal MR imaging morphometry by means of general pattern matching. Radiology 1996;199:787-91. Bibliographic Links| [Context Link] 
8. Fox PT, Perlmutter JS, Raichle ME. A stereotatic method of anatomical localization for positron emission tomography. J Comput Assist Tomogr 1985;9:141-53. [Context Link]

9. Minoshima S, Koeppe RA, Frey KA, Kuhl DE. Anatomic standardization: linear scaling and nonlinear warping of functional brain images. J Nucl Med 1994;35:1528-37. [Context Link]

10. Gee JC, Reivich M, Bajcsy R. Elastically deforming 3D atlas to match anatomical brain images. J Comput Assist Tomogr 1993;17:225-36. [Context Link]

11. Lin K-P, Huang S-C. An elastic mapping technique for intersubject tomographic image registration. Proc SPIE $1995 ; 2501: 938-50$. [Context Link]

12. Woods RP, Grafton ST, Holmes CJ, Cherry SR, Mazziotta JC. Automated image registration: I. General methods and intrasubject, intramodality validation. J Comput Assist Tomogr 1998;22:141-54. [Context Link]

13. Woods RP, Cherry SR, Mazziotta JC. Rapid automated algorithm for aligning and reslicing PET images. J Comput Assist Tomogr 1992;16:620-33. [Context Link]

14. Woods RP, Mazziotta JC, Cherry SR, MRI-PET registration with automated algorithm. J Comput Assist Tomogr 1993;17:536-46. [Context Link]

15. Woods RP, Mazziotta JC, Cherry SR. Automated image registration. In: Uemura K. Lassen NA, Jones T, Kanno I, eds.Quantification of brain function: tracer kinetics and image analysis in brain PET. Amsterdam: Excerpta Medica, $1993 ; 391-8$. [Context Link]

16. Talairach J, Tournoux P. Co-planar stereotaxic atlas of the human brain. Stuttgart: Thieme Medical, 1988. [Context Link]

17. Collins DL, Neelin P, Peters TM, Evans AC. Automatic 3D intersubject registration of MR volumetric data in standardized Talairach space. J Comput Assist Tomogr 1994;18:192-205. [Context Link]

18. Karcher H. Riemannian center of mass and mollifier smoothing. Commun Pure Appl Math 1977;30:509-41. [Context Link]

\section{APPENDIX}

\section{Method for Averaging Linear Spatial Transformations}

Given a set of linear transformations for aligning a group of individual brain images to a common reference, a method is described here for defining the average transformation from the individuals to the reference. Inverting this average transformation effectively defines a mapping from the common reference to a theoretical average brain that has shape, size, and orientation that are intermediate to those of the original brain images. Direct transformations from each original image to this theoretical average can then be defined using simple linear algebra.

A meaningful average transformation cannot be derived by simply averaging the elements of the individual transformation matrices. Proper averaging should preserve common attributes shared by the individual transformations(e.g., the average of a series of rigid body transformations should itself be a rigid body transformation), and quantifiable geometric attributes that vary across individuals should have intermediate values in the average. Fully rigorous mathematical methods exist for defining average geometric transformations (18) but require that differences between transformations be formally quantifiable as a metric on a multidimensional Riemannian manifold. Currently lacking this formal metric, a provisional approximation that meets the general criteria outlined above has proved to be sufficient for reconciling the Talairach transformation model and the resulting coordinate system with other linear transformation models.

The approximate method is based on the fact that a linear transformation for mapping image A to image B can be broken down into an arbitrary number of identical smaller linear transformations by computing the appropriate positive root of the original transformation. These smaller transformations preserve the geometric properties of the original. For example, computing the square root of a rigid body transformation from image $A$ to image $B$ defines a rigid body mapping from image $A$ to a position halfway to that of image $B$ in the sense that a second application of this same transformation will complete the mapping to image $B$.

Given linear transformations from images $A$ and $B$ to a common reference, the direct transformation from image $A$ to image $B$ can be derived by simple linear algebra. The square root of this direct transformation defines an average space with properties midway between those of the original two images. The transformation from this average space to the original common reference can be computed using linear algebra. A third image can be incorporated into the average by 
analogously averaging this first result with the new transformation for image $C$. In this case, the first value, which represents the cumulated mean of two of the original transformations, should be weighted twice as heavily when generating the new average. This is accomplished by computing the cube root of the derived direct transformation instead of the square root. This method can be generalized to average any arbitrary number of initial transformations to a common reference. The order in which the transformations are incorporated does change the result, but in the current context, differences between different orderings are negligible.

In addition to being used for defining an average brain size, shape, and orientation, this matrix averaging procedure can be used as part of a procedure for reconciling differences among all pairwise registrations of a set of images. If the direct registration of image $A$ to image $B$ is averaged together with all indirect transformation matrices derived by combining transformations for registering image $A$ to any other image $X$ and for registering that same image $X$ to image $B$, iterative replacement of the original transformations with the corresponding averages will eventually lead to convergence on a set of internally consistent transformations. In fact, this method was provisionally used to define the average rigid body transformations for the intrasubject validation study reported separately (12). After changing to the fully rigorous method(applicable only to rigid body transformations) described in that study, the two different methods were compared and found never to differ from one another by more than $1 \mu \mathrm{m}$.

Implementations of the approximate method for defining average brain size, shape, and orientation and the corresponding method for reconciling pairwise discrepancies to define mean transformations are included in the AIR 3.0 package. [Context Link]

Index Terms: Image registration; Magnetic resonance imaging; Emission computed tomography (PET); Brain mapping; Image warping

Accession Number: 00004728-199801000-00028

Copyright (c) 2000-2007 Ovid Technologies, Inc.

Version: rel10.5.2, SourceID 1.13281.2.32.1.0.1.96.1.3 